\title{
Four cases of coronavirus disease 2019 in the early stage of pandemic of South Korea: a single public hospital experience
}

Mi Young Ahn ${ }^{1,}$, Dong Hyun $\mathrm{Oh}^{1,}$, , Suhyun $\mathrm{Kim}^{1}$, Jae-Phil Choi ${ }^{1}$, Ji Hyeon Lee ${ }^{2}$, Young Kyung Lee ${ }^{3}$, and $\mathrm{Ki}$ Ho Hong ${ }^{4}$

Departments of ${ }^{1}$ Internal Medicine, ${ }^{2}$ Nursing, ${ }^{3}$ Radiology, and ${ }^{4}$ Laboratory Medicine, Seoul Medical Center, Seoul, Korea

Received: March 1, 2020

Revised : June 8, 2020

Accepted: June 18, 2020

\section{Correspondence to}

Ki Ho Hong, M.D.

Department of Laboratory

Medicine, Seoul Medical Center,

156 Sinnae-ro, Jungnang-gu,

Seoul 02053, Korea

Tel: +82-2-2276-7301

Fax: +82-2-2276-7302

E-mail: kihohongmd@gmail.com https://orcid.org/0000-0002-57009036

*'These authors contributed equally to this work.
In view of this pandemic, as of February 2020, South Korea has the second highest number of confirmed cases in the world. Herein, we report four confirmed coronavirus disease 2019 (COVID-19) cases in the early stage of the pandemic in South Korea and describe the identification, diagnosis, clinical course, and management, including one patient's initial mild symptoms at presentation and their progression to pneumonia on day 21 of illness. Within 48 hours of hospitalization, all four patients underwent evaluation for initial laboratory parameters, COVID-19 polymerase chain reaction (PCR), and chest computed tomography (CT) findings. All four mild COVID-19 patients were discharged, and they were re-examined 14 days after discharge. Despite all four of them being asymptomatic, one patient was re-admitted after confirmation of COVID-19 through PCR viral nucleic acid detection. She could be discharged after 7 days with two subsequent negative COVID-19 PCR at 24-hour intervals. Patients with mild COVID-19 generally have normal follow-up chest CT scans after discharge, even if the early chest CT definitely indicates pneumonia. Re-hospitalized patients with COVID-19 PCR positive results after discharge were not related to her initial chest CT, lab, symptoms compared other three patients.

Keywords: Coronavirus; Pandemics; Severe acute respiratory syndrome coronavirus 2
As of February 5, 2020, there were 24,623 confirmed cases of coronavirus disease 2019 (COVID-19) worldwide [1]. As of February 5, 2020, South Korea, a country that interacts heavily with China, had a total of 21 confirmed cases [2], the second highest number of confirmed cases in the world. Korea is one of the countries with substantial population migration, as it is geographically and economically close to China [2].

We report four confirmed cases with COVID-19 in the early stage of the pandemic (the 5th, $7^{\text {th }}$, 9th, and 19th patient cases) in South Korea. We collected data and ra- diologic images from electronic medical records of the four patients with mild COVID-19 to assess each of the their clinical courses.

We performed real-time polymerase chain reaction (RT-PCR) to assess the presence of the severe acute respiratory syndrome coronavirus 2 (SARS-CoV-2) E gene and the $R d R p$ gene from nasopharyngeal swabs, oropharyngeal swabs, and sputum collected from all four patients. PCR was performed with the PowerChek 2019nCoV Real-time PCR Kit (Kogene Biotech, Seoul, Korea). This kit is the first product to be approved by the Ko- 

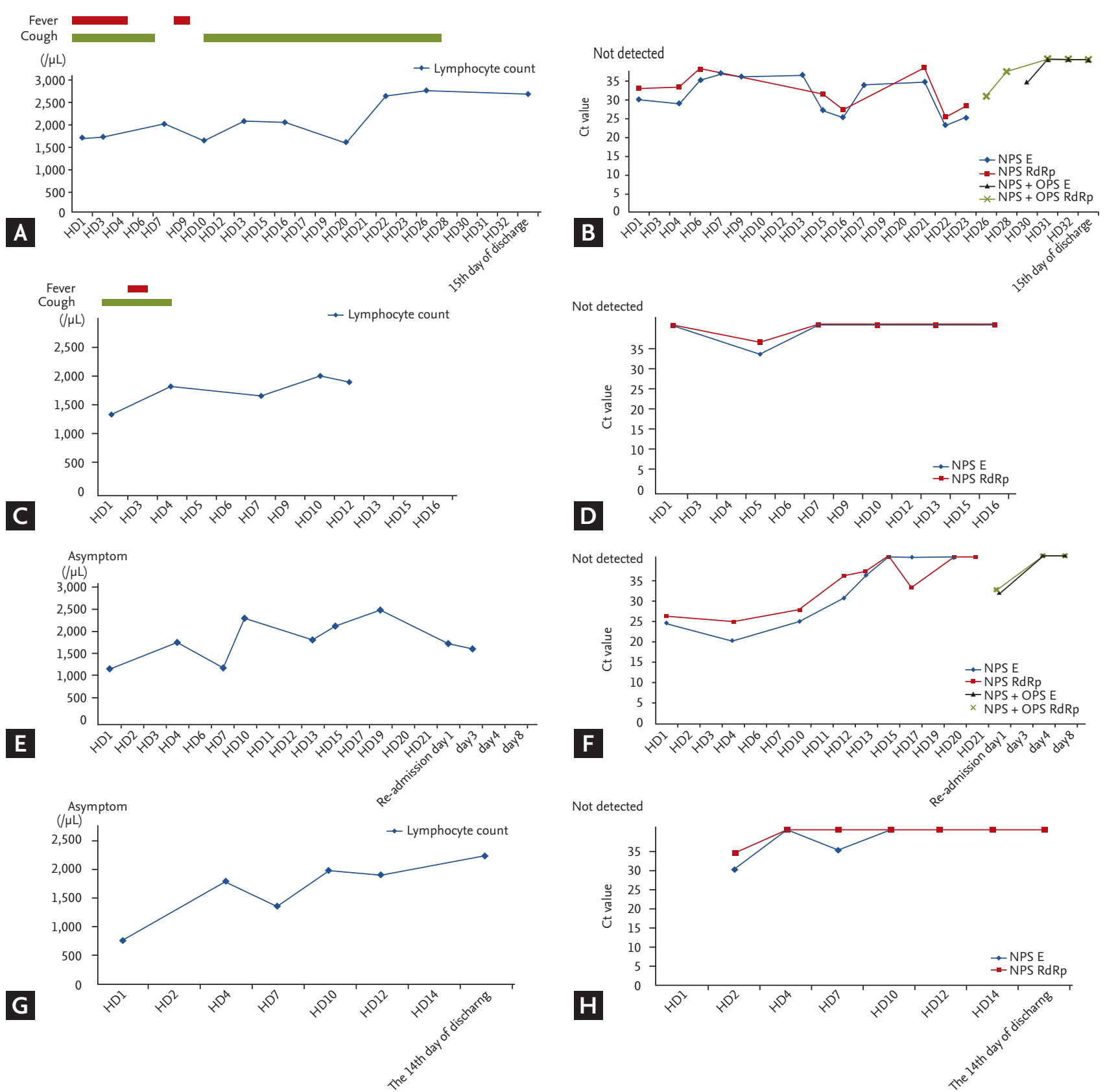

Figure 1. Absolute lymphocyte count of all four cases were decreased at first, but it shows "wax and wane," and then recovered. (A, B) Case 1 (patient no. 5 of domestic confirmed cases), for a relatively long period of time, the upper and lower grades were continuously positive. Within 7 days of onset, cycle threshold (Ct) was lower in the sputum, but was better detected in the upper respiratory specimen after 7 days. (C, D) Case 2 (patient no. 7 of domestic confirmed cases) continued to show a Ct value close to the threshold value except at onset, and positive results observed only for the sputum. (E, F) Case 3 (patient no. 9 of domestic confirmed cases) was a close contact of Case 1 and did not experience clinical symptoms throughout the hospital course. The virus was detected for a relatively long time in the upper respiratory specimen and has not been detected for 10 days in the sputum. The Ct value is also slightly lower in the upper respiratory specimen, suggesting that the virus titer is likely to be higher. (G, H) Case 4 (patient no. 19 of domestic confirmed cases) was positive for the virus in the initial specimen obtained on hospital day 1 (HD1). However, polymerase chain reaction (PCR) results from follow-up specimens have never fulfilled PCR positivity criteria (positive for both genes), suggesting very low virus titers around threshold values. In view of these results, it may be helpful to diagnose the sample type mentioned at the onset of symptoms, and the use additional samples taking into account the patient's symptoms and imaging findings. During recovery, detection of the virus in upper-altitude samples appears to last longer than detection in the lower-altitude samples. NPS, nasopharyngeal swab; RdRp, RNA dependent RNA polymerase gene; OPS, oropharyngeal swab. 
rean Food and Drug Adminstration for the in vitro diagnosis of SARS-CoV-2, having received emergency use authorization in Korea on February 7, 2020. It is based on the protocol suggested by Corman et al. [3] and recommended by the World Health Organization. We also performed PCR assay with this kit for samples collected before February 7, 2020. If the cycle threshold (Ct) values were $\leq 35$ for both the $E$ and RdRp genes, then the test result was considered positive for SARS-CoV-2. The PCR results are shown in Fig. 1

Case 1 was the 5 th patient diagnosed in South Korea. On January 29, 2020, a 32-year-old man presented with a 2-day history of cough and subjective fever. He had returned to Seoul on January 24 after a business trip to Wuhan, China.

Apart from a history of bronchial asthma, controlled by a low-dose inhaled corticosteroid/long-acting beta-agonist, the patient was an otherwise healthy non-smoker. Physical examination revealed a body temperature of $36.6^{\circ} \mathrm{C}$, blood pressure of $134 / 75 \mathrm{mmHg}$, pulse of 78 beats per minute, respiratory rate of 11 breaths per minute, and oxygen saturation of $100 \%$ while the patient was breathing ambient air. Lung auscultation revealed no adventitious sounds including crackle or wheezing. A nasopharyngeal swab specimen was obtained and sent for detection of viral respiratory pathogens by nucleic acid amplification testing; the results were reported within 48 hours as negative for all pathogens tested, including influenza A and B, parainfluenza, respiratory syncytial virus, metapneumovirus, rhinovirus, adenovirus, and three common coronavirus strains known to cause illness in humans (NL63, 229E, and OC43).

Initial chest radiography performed on hospital day (HD) 1 showed focal ill-defined increased opacity in the upper lobe of the left lung (Supplementary Fig. 1A).

The patient's body temperature increased to $37.6^{\circ} \mathrm{C}$ on the day of hospitalization but improved on its own. On $\mathrm{HD} 2$, his highest temperature was $38.3^{\circ} \mathrm{C}$, and his highest temperature was $37.5^{\circ} \mathrm{C}$ on $\mathrm{HD}_{3}$. His temperature was checked on $\mathrm{HD}_{5}$, and from HD6, he was without definite fever, until HD23. The patient continued to report a nonproductive or productive cough. The patient complained of a productive cough up until $\mathrm{HD}_{3}$, and thereafter, a mild nonproductive cough waxed and waned, which was not aggravated compared to that before admission. His respiratory symptoms were controlled with inhaled corticosteroid/long-acting beta-agonist and antitussive agents with expectorants on demand. His initial low-dose chest axial computed tomography (CT) scan obtained on the same HD showed multifocal ill-defined ground glass opacities (GGOs) in both lower lobes, mainly distributed along the bronchovascular bundles and subpleural regions (Supplementary Fig. ${ }_{1 B}$ and ${ }_{1 C}$ ). Given the CT findings suggestive of viral pneumonia, treatment with lopinavir/ritonavir (Kaletra, AbbVie, North Chicago, IL, USA) was initiated on HD2 and maintained for 14 days. Additionally, we empirically prescribed moxifloxacin $400 \mathrm{mg} /$ day for 1 week.

Case 2 was the 7 th patient diagnosed in South Korea. On January 29, 2020, a 28-year-old man with a 5-day history of cough and subjective fever had returned to Seoul on January 23 after a trip to Wuhan, China. The patient stated that he had fever and fatigue since the evening of January 25; on January 28, he developed fever and cough with sputum. On January 29, he needed antipyretics and therefore underwent testing for SARS-CoV-2. He was healthy with no past medical history and was a social drinker and a nonsmoker. Physical examination revealed a body temperature of $37.7^{\circ} \mathrm{C}$, blood pressure of $146 / 88 \mathrm{mmHg}$, pulse of 90 beats per minute, respiratory rate of 18 breaths per minute, and oxygen saturation of $98 \%$ while the patient was breathing ambient air.

On admission, the patient reported a persistent productive cough and a 2-day history of fever and no shortness of breath or chest pain. Vital signs were within normal ranges, except the body temperature of $37.7^{\circ} \mathrm{C}$.

There was no further fever within the hospitalization period. Initial chest radiography obtained on $\mathrm{HD} 1$ showed ill-defined increased opacity in the upper lobe of the left lung (Supplementary Fig. 2A). An initial low-dose chest axial CT scan obtained on HD2 showed peribronchial ill-defined consolidation with peripheral GGOs in the left upper lobe (Supplementary Fig. 2B), and we started lopinavir/ritonavir (Kaletra) and empirical antibiotic treatment. We administered lopinavir/ritonavir for 2 weeks without other side effects. His symptoms improved immediately after admission; hence, he was not placed on antipyretics or other symptomatic medication. Chest radiography obtained on $\mathrm{HD}_{3}$ revealed a slightly increased extent of the focal ill-defined opacities in the left upper lung zone, and wax-and-waning pattern abnormalities during the hospital stay thereafter. How- 
ever, imaging study after HD12 showed improvement of the increased opacity in the left upper lung zone, laboratory results on $\mathrm{HDs}_{3}$ and $\mathrm{HD}_{7}$ indicated leukopenia, mild thrombocytopenia, and elevated levels of LDH

Case 3 was the 9th patient diagnosed in South Korea. On January 29, 2020, a 27-year-old woman presented with a subjective fever; she met frequently with the patient mentioned in Case 1 between January 25 and January 29, 2020, but she had no symptoms.

She did not have a past medical history and was a healthy nonsmoker. Physical examination revealed a body temperature of $36.4^{\circ} \mathrm{C}$, blood pressure of $148 / 118$ $\mathrm{mmHg}$, pulse of 87 beats per minute, respiratory rate of 21 breaths per minute, and oxygen saturation of $98 \%$ while the patient was breathing ambient air. Initial chest radiography obtained on $\mathrm{HD} 1$ showed ill-defined increased opacity in the left upper lung zone (Supplementary Fig. 3A). Initial low-dose chest axial CT scan obtained on $\mathrm{HD}$ s showed peribronchial consolidation with a relatively well-defined margin in the left lower lobe (Supplementary Fig. 3B). On admission, she reported no subjective symptoms such as fever, cough, or sputum; instead, she experienced depression and acute stress reaction. We started lopinavir $400 \mathrm{mg} /$ ritonavir $100 \mathrm{mg}$ and empirical antibiotic treatment, based on her chest CT scan findings. The patient did not require oxygen throughout the hospital stay and she was stable without respiratory symptoms or fever; therefore, a 2-week medication period was planned. However, on HDio, the medication was stopped because of an increase in triglyceride level to $921 \mathrm{mg} / \mathrm{dL}$. On HDio, premature ventricular contraction (PVC) was checked by heart rate monitoring, and the electrocardiogram (ECG) showed 10 to 15 bigeminy per minute. Cardiology consultation was performed, and the probable cause determined was the antibiotics and antiviral drug previously used. On HD23, her ECG monitoring showed two PVC findings per minute. The 27-year-old young female patient did not need fluid treatment or antipyretics. Only empirical antibiotics and antiviral drugs were administered.

Case 4 was the 19th patient diagnosed in South Korea. On February 4, 2020, a 36-year-old man with subjective fever contacted the health center and was admitted to the main hospital on February 5, 2020. He had no past medical history and was a healthy nonsmoker who did not drink alcohol. He worked with a company in an of- fice building. Physical examination revealed a body temperature of $37.7^{\circ} \mathrm{C}$, blood pressure of $141 / 92 \mathrm{mmHg}$, pulse of 85 beats per minute, respiratory rate of 18 breaths per minute, and oxygen saturation of $95 \%$ while the patient was breathing ambient air.

On admission, he had no respiratory symptoms. Initial chest radiography obtained on HDi showed an ill-defined increased opacity in the right lower lung zone (Supplementary Fig. 4A). An initial low-dose chest axial CT scan obtained on $\mathrm{HD} 2$ showed multiple centrilobular nodules with peripheral GGOs in the right lower lobe (Supplementary Fig. 4B). He was administered lopinavir $400 \mathrm{mg} /$ ritonavir $100 \mathrm{mg}$ for 14 days and had no complaints of respiratory symptoms, except for mild diarrhea after starting the medication. Apart from the patient's consistent subclinical symptoms, his early chest radiograph revealed increased severity of the peribronchial ill-defined opacities in the right middle lung zone and the right lower lung zone, until HD4. Chest radiography obtained on $\mathrm{HD} 15$ showed decreased severity of the peribronchial ill-defined opacities in the right lower lung zone and he was discharged on $\mathrm{HD} 16$.

Complete blood counts and serum chemical studies were available starting on HD1. Laboratory results on $\mathrm{HD}_{1}, \mathrm{HD}_{4}$, and $\mathrm{HD}_{7}$ indicated leukopenia. In addition, there were no alterations in hepatic function measures.

All four patients admitted to the hospital were in their late $20 \mathrm{~s}$ and mid-3os, all minor; none of them needed oxygen, unlike the cases reported in China and other large-scale studies. The initial chest X-ray showed a minor pneumonia finding that a general doctor might miss. In the case of the main hospital, the chest CT was filmed with closed movement lines both on the day of hospitalization or on the next day, and the CT findings were typical of worse-than-expected viral pneumonia. Generally, four patients like in this case would not have undergone chest CT if they had visited the hospital and had initial laboratory, chest X-ray, and clinical symptoms. However, reports from China have suggested poor outcomes and death in approximately $10 \%$ of patients when they showed rapid chest X-ray deterioration; therefore, a chest CT scan is advisable [4-6].

Lopinavir/ritonavir (Kaletra) are expected to prevent early viral replication through a protease inhibitor, and it is thought that additional benefits would be a reduction in the transmission rate to the clinical workforce 
by lowering the patient's viral load [7], even though it is already proven to be ineffective.

All four patients showed subtle abnormalities in the chest radiography, and minimal respiratory symptoms; however, their chest CT scans were typical for viral pneumonia. Typical CT patterns of viral pneumonia are (1) multifocal bronchopneumonia, (2) organizing pneumonia, and (3) diffuse alveolar damage patterns [8]. Of these patterns, all four cases showed multifocal bronchopneumonia patterns with mild extent, and gradual radiological improvement eventually; neither pleural effusion nor lymphadenopathy was found. The findings of the CT scan of the first case were multiple GGOs only, without consolidation. The radiographical abnormalities for the other three cases indicated a mixed pattern of GGO and consolidation. Only Case 2 demonstrated upper lung zone involvement, while the other cases showed lower lung zone predominance. The extent of the lung involvement was below $20 \%$, which was consistent with mild pneumonia. Through follow-up of the serial chest radiograph, only Case 4 showed aggravated radiological abnormalities in the early phase up to $\mathrm{HD}_{5}$; however, the patient presented only with minimal symptoms such as transient chilling, which had resolved before admission. He was discharged on $\mathrm{HD} 14$ with negative conversion of SARS-CoV-2 PCR and with greatly improved chest radiography.

The main role of imaging in viral infection lies in the detection or exclusion of pneumonia findings, as chest radiographies are normal or inconclusive [8]. The simple radiologic manifestations of viral pneumonia are nonspecific and difficult to differentiate from those of other infections, as seen in our four cases. Furthermore, because it is difficult to determine whether or not pneumonia is present from the symptoms and lab tests alone, it is necessary to anticipate the possibility of pneumonia and monitor it carefully, using early chest CT scans if available.

COVID-19 patients are reported to generally develop pneumonia around the end of the first week to the beginning of the second week of illness, like those with SARS [9]. Therefore, it will be necessary to understand and cope with the severity and pneumonia that may deteriorate, or improve, depending on the timing of the diagnosis with regard to the onset.

\section{Conflict of interest}

No potential conflict of interest relevant to this article was reported.

\section{REFERENCES}

1. Korea Ministry of Health and Welfare. Trend of occurrence COVID-19 in South Korea [Internet]. Sejong (KR): MOHW, 2020 [cited 2020 Jun 23]. Available from: http:// ncov.mohw.go.kr/bdBoardList_Real.do?brdId=\&brdGubun=\&ncvContSeq=\&contSeq=\&board_id=\&gubun=.

2. World Health Organization. Coronavirus disease 2019 (COVID-19) Situation Report 38 [Internet]. Geneva (CH): WHO, 2020. [cited 2020 Jun 23]. Available from: https:// www.who.int/docs/default-source/coronaviruse/situation-reports/20200227-sitrep-38-covid-19.pdf.

3. Corman VM, Landt O, Kaiser M, et al. Detection of 2019 novel coronavirus (2019-nCoV) by real-time RT-PCR. Euro Surveill 2020;25:2000045.

4. Wang $\mathrm{D}, \mathrm{Hu} \mathrm{B}, \mathrm{Hu} \mathrm{C}$, et al. Clinical characteristics of 138 hospitalized patients with 2019 novel coronavirus-infected pneumonia in Wuhan, China. JAMA 2020;323:10611069.

5. Huang C, Wang Y, Li X, et al. Clinical features of patients infected with 2019 novel coronavirus in Wuhan, China. Lancet 2020;395:497-506.

6. Chen N, Zhou M, Dong X, et al. Epidemiological and clinical characteristics of 99 cases of 2019 novel coronavirus pneumonia in Wuhan, China: a descriptive study. Lancet 2020;395:507-513.

7. Arabi YM, Alothman A, Balkhy HH, et al. Treatment of Middle East Respiratory Syndrome with a combination of lopinavir-ritonavir and interferon- $\beta 1 \mathrm{~b}$ (MIRACLE trial): study protocol for a randomized controlled trial. Trials 2018;19:81.

8. Lee KS. Pneumonia associated with 2019 novel coronavirus: can computed tomographic findings help predict the prognosis of the disease? Korean J Radiol 2020;21:257-258.

9. Wong JEL, Leo YS, Tan CC. COVID-19 in Singapore-current experience: critical global issues that require attention and action. JAMA 2020 Feb 20 [Epub]. https://doi. org/10.1001/jama.2020.2467. 

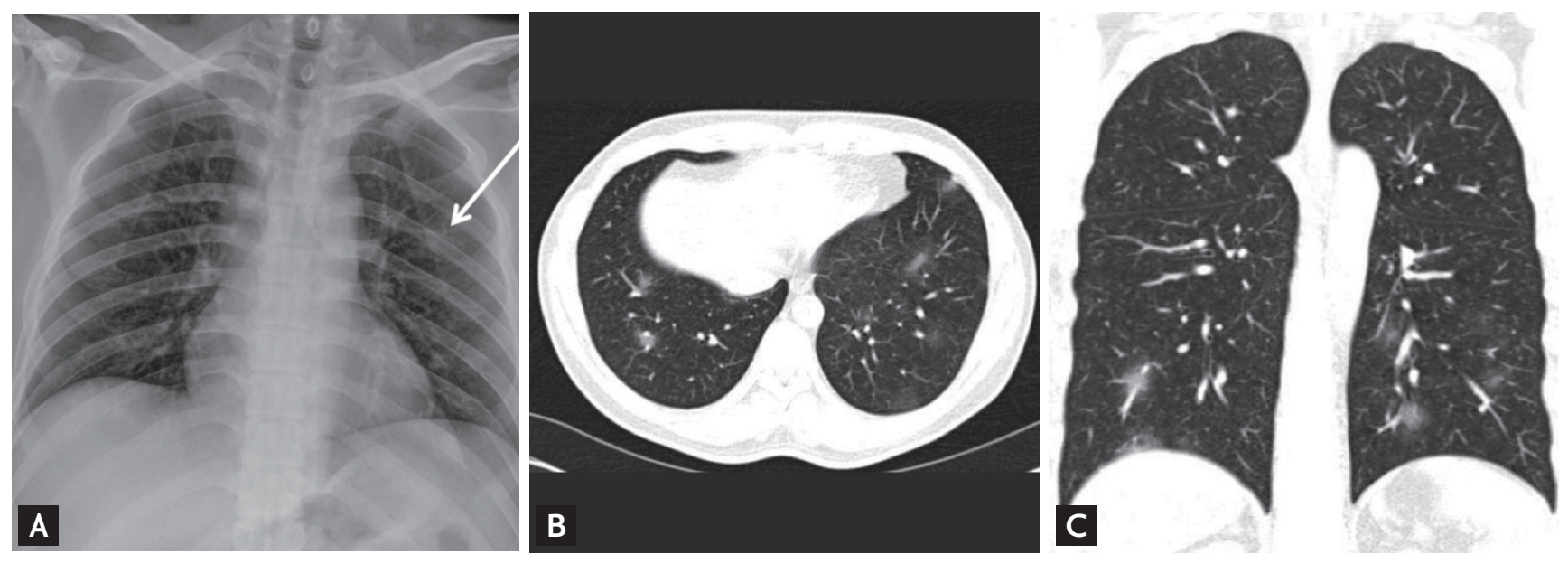

Supplementary Figure 1. Case 1. A 32-year-old man diagnosed with coronavirus disease 2019 pneumonia without secondary infection. (A) Initial chest radiography obtained on the 1st hospital day shows the focal ill-defined increased opacity in left upper lung zone (arrow). (B) Initial low dose chest axial computed tomography (CT) scan obtained on the 2 nd hospital day shows multifocal ill-defined ground glass opacities (GGOs) in both lower lobes, mainly distributed along the bronchovascular bundles and subpleural regions. (C) Initial low dose chest coronal CT scan obtained on the 2nd hospital day shows multifocal ill-defined GGOs along the bronchovascular bundles in both lower lobes. 

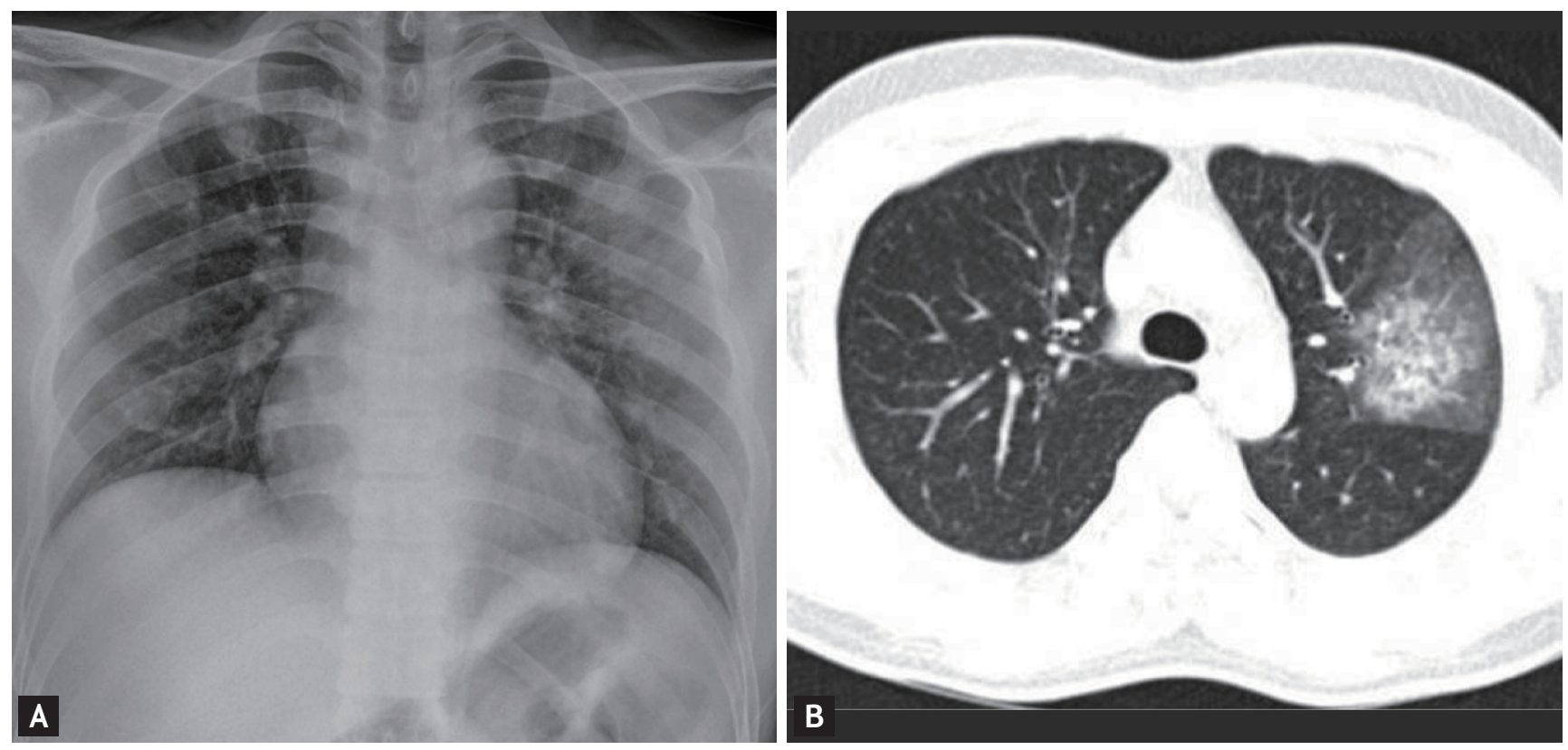

Supplementary Figure 2. Case 2. A 28-year-old man diagnosed with coronavirus disease 2019 pneumonia without secondary infection. (A) Initial chest radiography obtained on the 1st hospital day shows the ill-defined increased opacity in the left upper lung zone. (B) Initial low dose chest axial computed tomography scan obtained on the 1st hospital day shows peribronchial ill-defined consolidation with peripheral ground glass opacities in the left upper lobe. 

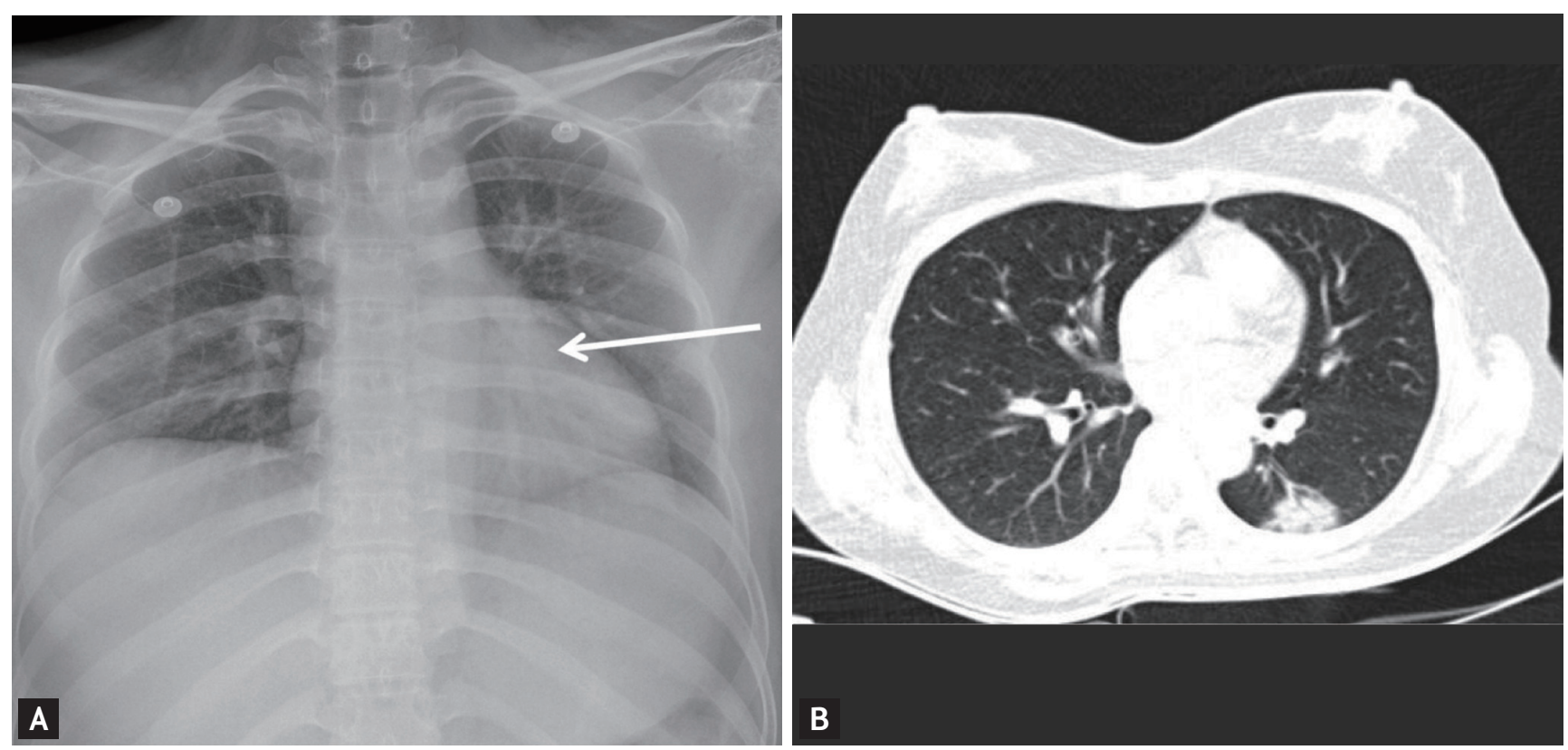

Supplementary Figure 3. Case 3. A 28-year-old woman diagnosed with coronavirus disease 2019 pneumonia without secondary infection. (A) Initial chest radiography obtained on the 1st hospital day shows the ill-defined increased opacity in the left upper lung zone (arrow). (B) Initial low dose chest axial computed tomography scan obtained on the ist hospital day shows peribronchial consolidation with a relatively well-defined margin in the left lower lobe. 

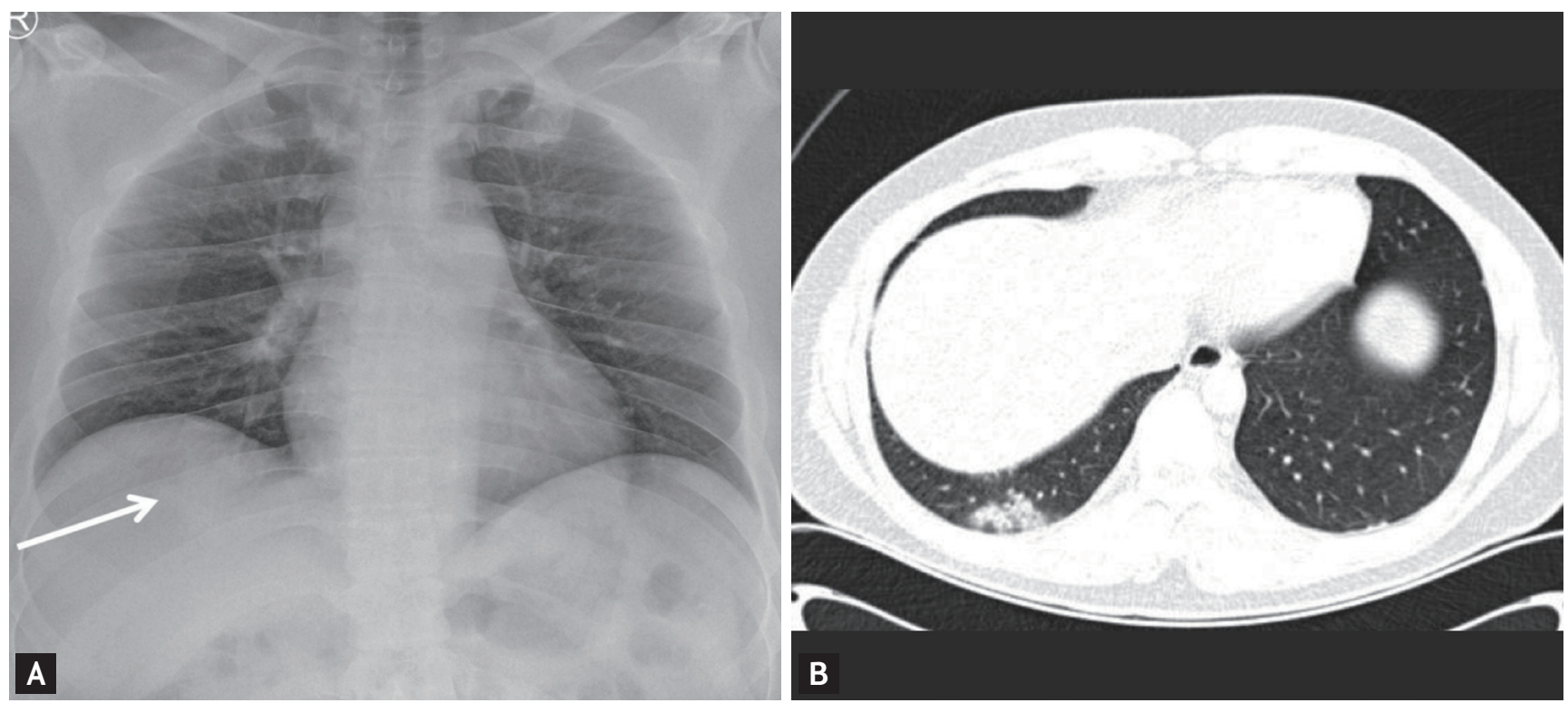

Supplementary Figure 4. Case 4. A 36-year-old man diagnosed with coronavirus disease 2019 pneumonia without secondary infection. (A) Initial chest radiography obtained on the 1st hospital day shows the ill-defined increased opacity in the right lower lung zone (arrow). (B) Initial low dose chest axial computed tomography scan obtained on the 2nd hospital day shows multiple centrilobular nodules with peripheral ground glass opacities in the right lower lobe. 